\title{
Long Range Potential Effects in Low Density Krypton Gas
}

\author{
F. Formisano, ${ }^{1,2}$ C. J. Benmore,${ }^{3}$ U. Bafile,${ }^{4}$ F. Barocchi,,${ }^{1,2}$ P. A. Egelstaff, ${ }^{3}$ R. Magli,${ }^{5,2}$ and P. Verkerk ${ }^{6}$ \\ ${ }^{1}$ Dipartimento di Fisica, Università degli Studi di Firenze, Largo E. Fermi 2, I-50125 Firenze, Italy \\ ${ }^{2}$ Istituto Nazionale di Fisica della Materia, Sezione di Firenze, Largo E. Fermi 2, I-50125 Firenze, Italy \\ ${ }^{3}$ Physics Department, University of Guelph, Ontario, Canada N1G 2 W1 \\ ${ }^{4}$ Istituto di Elettronica Quantistica, Consiglio Nazionale delle Ricerche, Via Panciatichi 56/30, I-50127 Firenze, Italy \\ ${ }^{5}$ Dipartimento di Energetica “S. Stecco," Università degli Studi di Firenze, Via di S. Marta 3, I-50139 Firenze, Italy \\ ${ }^{6}$ Interfacultair Reactor Instituut, Technische Universiteit Delft, 2629 JB Delft, The Netherlands
}

(Received 12 November 1996)

Small angle neutron scattering (SANS) in low density ${ }^{86} \mathrm{Kr}$ gas has been performed in order to measure the small- $k$ behavior of the static structure factor $S(k)$. Three number densities between 1.52 and $2.42 \mathrm{~nm}^{-3}$ along the $T=297 \mathrm{~K}$ isotherm have been studied. The small- $k$ dependence of the Fourier transform $c(k)$ of the direct correlation function $c(r)$ has been derived. The experimental determination of the $k^{3}$ term in the behavior of $c(k)$ has led to a direct measurement of the London dispersion interaction in the pair potential of krypton. Also the contribution of the three-body potential in the asymptotic behavior of $c(r)$ has been observed and related to the magnitude of the three-body interaction potential. [S0031-9007(97)03538-2]

PACS numbers: 51.90.+r, 34.20.Cf, 61.12.Ex

The London dispersion energy due to the induced dipole interactions determines the form of the long range potential of pairs, as well as the form of the long range irreducible three-body interaction in ground state atoms $[1,2]$. One possibility for a direct assessment of these long range interactions among the particles in a fluid is given by the connection between the small- $k$ behavior of the static structure factor $S(k)$ [Eq. (1) below] and the long range microscopic forces. This connection has been pointed out by Enderby, Gaskell, and March [3], who emphasized the importance of direct observation of such terms. Several papers have been devoted to this subject [4-6], and recently this matter has been discussed by Reatto and Tau [7]. The main result of these papers is that for classical fluid insulators, like noble gases, $S(k)$ and $c(k)$ [Eq. (2) below], which is the Fourier transform of the direct correlation function $c(r)$, are expected to have at small $k$ a nonanalytic $k^{3}$ term directly related to the $r^{-6}$ tail of the microscopic van der Waals interaction potential in the fluid. In particular, the theory of Reatto and Tau [7] takes into account the effect of retardation which modifies $k^{3}$ to $k^{4}$ as $k \rightarrow 0$. Moreover, they confirm that measurements of the $k^{3}$ behavior of $c(k)$ in low density gases can, in principle, give an experimental direct verification of the $r^{-6}$ power law of the pair potential and might determine the long range behavior of the dressed three-body vertex. However, we shall show that in the present case the $k^{2}$ term includes a larger and more useful measure of the dressed three-body vertex, giving a direct and unique experimental access to the long range interaction.

Neutron diffraction measurements, when possible with high accuracy, have been proven to be a direct method for the determination of the interaction potential between pairs of atoms in gases [8,9]. Recently, both normal diffraction and SANS experiments have been performed in argon gas in order to measure the $k^{3}$ dependence of $c(k)$ and give an experimental value of the amplitude $B$ of the long range $-B / r^{6}$ pair potential $[10,12]$ in agreement with that determined by more traditional methods. Here we report the results of the measurements of the small$k$ dependence of the $c(k)$ of krypton at low density from which the strength $B$ is measured together with the approximate effect of the three-body forces on the asymptotic behavior of $c(r)$. This is the first direct detection of this effect. Krypton has been chosen in this case because the strength of the three-body potential is larger than in argon, where the effect of the Axilrod and Teller (AT) three-body potential [12] could not be detected [11].

By means of theoretical calculations of $c(k)$, performed with the modified hypernetted chain equation, Reatto and Tau [7] have shown that in low density noble gases, the $k$ range useful for the determination of the $k^{3}$ dependence is $0.5<k<3.5 \mathrm{~nm}^{-1}$. This range is experimentally accessible with modern SANS instrumentation. The lower limit is determined by retardation effects and the upper limit by higher order terms. Our measurements have been performed with the PAXE diffractometer at the Laboratoire Léon Brillouin in Saclay. An important innovation was to use the isotope ${ }^{86} \mathrm{Kr}$ in the neutron experiment in order to minimize contributions from nuclear absorption and incoherent scattering. The neutron cross sections of ${ }^{86} \mathrm{Kr}$ are $\sigma_{\mathrm{coh}}=8.2(5), \sigma_{\mathrm{inc}}=0$, and $\sigma_{\mathrm{abs}}=0.003$ (barn), for the coherent, incoherent, and absorption cross sections, respectively [13].

In the theory of simple fluids, the static structure factor $S(k)$ and the Fourier transform $c(k)$ of the direct 
correlation function $c(r)$ are defined by

$$
\begin{gathered}
S(k)=1+n \int d \boldsymbol{r} \exp (-i \boldsymbol{k} \cdot \boldsymbol{r})[g(r)-1], \\
c(k)=\int d \boldsymbol{r} \exp (-i \boldsymbol{k} \cdot \boldsymbol{r}) c(r),
\end{gathered}
$$

where $n$ is the number density, $g(r)$ is the pair correlation function, and $c(r)$ is given by the Ornstein-Zernike relation

$$
g(r)-1 \equiv h(r)=c(r)+n \int d \boldsymbol{r}^{\prime} c\left(r^{\prime}\right) h\left(\boldsymbol{r}-\boldsymbol{r}^{\prime}\right) .
$$

$S(k)$ is an experimentally accessible quantity and $c(k)$ can be derived from $S(k)$ by using the relationship obtained from Eqs. (1)-(3):

$$
c(k)=[S(k)-1] / n S(k) .
$$

The present experiment has been performed along the $297 \pm 1 \mathrm{~K}$ isotherm at a range of low densities, so that higher order many-body contributions were minimized as in previous experiments $[9,11,14]$. For the analysis in the present paper we use only the most accurate data at densities $n=1.52,1.97$, and $2.42 \mathrm{~nm}^{-3}$. The densities are determined with a relative accuracy of $0.5 \%$. The ${ }^{86} \mathrm{Kr}$ gas was kept in a flat cell of $3.48 \mathrm{~cm}$ thickness with single crystal sapphire windows to reduce container scattering and capable of withstanding more than 100 bars of pressure. The experiment was performed with a neutron wavelength of $4.0 \AA$, and the distance between the sample and the two-dimensional $\mathrm{BF}_{3}$ gas detector was $171.5 \pm 0.5 \mathrm{~cm}$. The choice of these values allowed the $0.5<k<4.3 \mathrm{~nm}^{-1}$ range to be covered with an overall resolution $\Delta k / k \sim 10 \%$.

A detailed description of the experimental procedure as well as of the data analysis will be given elsewhere. Here we mention only that the neutron intensity data were corrected for the effect of background, self-shielding, multiple and inelastic scattering, and detector efficiency, and normalized in absolute units to obtain $S(k)$. The data agreed with the compressibility limit at $S(k=0)$ (see Ref. [15]) to within $1.5 \%$. As a final adjustment we renormalized the $S(k)$ to the $k=0$ value calculated from $P V T$ data [15], which was equivalent to replacing 8.2 by 8.1 barns for the ${ }^{86} \mathrm{Kr}$ coherent cross section. We estimate that this data analysis procedure leads to a final uncertainty on the absolute scale of $S(k)$ of the order of $1 \%$. The final $c(k)$ 's were then derived from the $S(k)$ 's by means of Eq. (4). Figure 1 shows the $c(k)$ 's for ${ }^{86} \mathrm{Kr}$ at three measured densities, together with previous experimental data (measured for natural krypton) in the higher part of the $k$ range [14], and with experimental $c(k=0)$ values obtained by combining Eq. (4) with the thermodynamical data of Ref. [15]. The agreement between the two sets of experimental data is reasonable in the overlapping region.

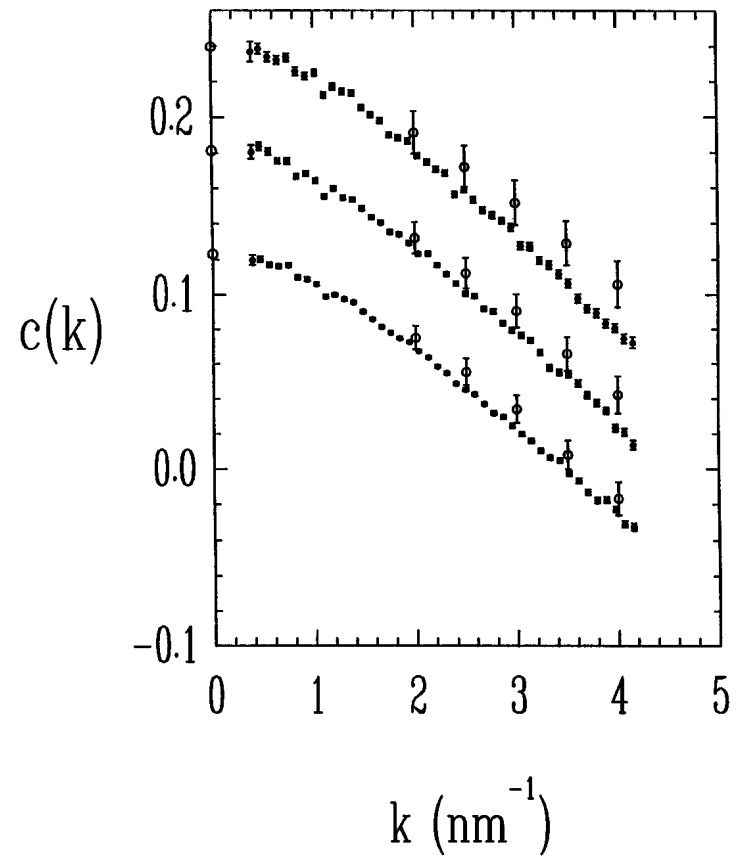

FIG. 1. The experimental $c(k)$ for krypton at $T=297 \mathrm{~K}$ is given by the filled circles. From top to bottom: $n=1.52$, 1.97, and $2.42 \mathrm{~nm}^{-3}$ (1.97 and $1.52 \mathrm{~nm}^{-3}$ data have been shifted upwards by 0.05 and 0.10 , respectively). The statistical uncertainties are within the size of the symbols. The open circles and error bars are the data of Ref. [14]. $k=0$ values are calculated from $P V T$ data [15].

In very low density systems the interaction law can be written retaining only the pair and triplet contributions and it has been demonstrated [7] that, within suitable approximations, the asymptotic behavior of $c(r)$ is given by

$$
c(r)=-\beta \phi(r)+C(r), \quad \text { as } r \rightarrow \infty,
$$

with

$$
\begin{aligned}
C(r)=n \int d & \boldsymbol{r}_{3} g\left(r_{13}\right) g\left(r_{23}\right) \\
& \times\left\{\exp \left[-\beta \phi_{3}\left(\boldsymbol{r}_{1}, \boldsymbol{r}_{2}, \boldsymbol{r}_{3}\right)\right]-1\right\},
\end{aligned}
$$

where $\beta=1 / k_{\mathrm{B}} T$, and $\phi(r), \phi_{3}$, and $C(r)$ are the pair, triplet potential, and dressed three particle vertex, respectively. The total interatomic interaction is given by

$$
u\left(\boldsymbol{r}_{1}, \boldsymbol{r}_{2}, \ldots, \boldsymbol{r}_{N}\right)=\sum_{i<j} \phi\left(r_{i j}\right)+\sum_{i<j<k} \phi_{3}\left(\boldsymbol{r}_{i}, \boldsymbol{r}_{j}, \boldsymbol{r}_{k}\right) .
$$

When the long range dispersion term is the dominant one we have

$$
\phi(r)=-B / r^{6}, \quad \text { as } r \rightarrow \infty .
$$

Moreover, if the irreducible three-body interaction is assumed to be of the AT form [12], it can be demonstrated $[7,16]$ that the asymptotic behavior of $C(r)$ is

$$
C(r)=-(8 \pi / 3) \beta \nu n / r^{6}, \quad \text { as } r \rightarrow \infty .
$$


Here $\nu$ is the strength of the AT potential. By using expressions (5) - (7) and asymptotic Fourier analysis [7] it can be shown that the small- $k$ expansion of $c(k)$ is given by

$$
c(k)=c(0)+c_{2} k^{2}+c_{3}\left|k^{3}\right|+c_{4} k^{4}+\cdots,
$$

where both $c_{2}$ and $c_{3}$ involve the two- and three-body potentials and $c_{4}$ and higher terms will be neglected. By comparing Eq. (9) of Ref. [17] with Eq. (5) of Ref. [7] it may be seen that both $c_{2}$ and $c_{3}$ involve the three-particle vertex of Eq. (6). After algebraic reduction a very simple result for $c_{3}$ is obtained [7] which is exact for systems with a pair plus $\mathrm{AT}$ interaction potential:

$$
c_{3}=\beta\left(\pi^{2} / 12\right)[B-(8 \pi / 3) \nu n],
$$

which depends linearly on the density due to the AT potential. In principle a determination of $c_{3}$ as the function of density would yield experimental values for $B$ as well as for $\nu$. In contrast, for $c_{2}$ no analytical expression is available. Note that $C(r)=0$ and $c_{3}$ is a constant as the function of density in the absence of irreducible long range three-body interactions.

In order to display the $k^{3}$ dependence of the experimental $c_{\text {exp }}(k)$ it is more convenient to plot the quantity $\lambda(k)$ defined as

$$
\lambda(k)=[c(k)-c(0)] / k^{2}=c_{2}+c_{3}|k|+\cdots .
$$

Over the range where $\lambda_{\exp }(k)$ is linear in $k$, we may neglect $c_{4}$ and higher terms in Eq. (9) (Reatto and Tau [7] estimate this range to be $\left.0.5<k<3.5 \mathrm{~nm}^{-1}\right)$. To derive the best data on $\lambda(k)$ we used two independent methods to evaluate it. In one we performed a least squares fit for the three parameters $c(0), c_{2}$, and $c_{3}$ in Eq. (9), and then calculated $\lambda(k)$ analytically. In the other we deduced $c(0)$ from the $P V T$ data [15] and evaluated $\lambda(k)$ for each experimental value of $c(k)$. There was excellent agreement between these two methods, and we noted that $\lambda(k)$ was a simple function, being nearly linear in $k$.

Figure 2 shows the experimental $\lambda_{\exp }(k)$ for krypton at $T=297 \mathrm{~K}$ for three different measured densities, together with the theoretical results for the low density expansion of $c(k)$ using the Aziz pair potential and the AT three-body interaction (Table 3 in Ref. [17]). From this figure the linear $k$ dependence of $\lambda_{\text {exp }}(k)$ for $k<3.5 \mathrm{~nm}^{-1}$ and therefore the $k^{3}$ dependence of $c_{\text {exp }}(k)$ [see Eq. (9)] can be observed. The experimentally observed near-linearity also demonstrates the approximate correctness of the long range behavior of $\phi(r)$ and of the three-body vertex $C(r)$ given in Eqs. (7) and (8). Adding the AT potential in the theoretical calculation of $\lambda(k)$ clearly changes $c_{2}$, but hardly $c_{3}$, which is not surprising since $B \gg 8 \pi \nu n / 3$ in the expression for $c_{3}$, Eq. (10) $\left(B=12.7 \times 10^{-24} \mathrm{~J} \mathrm{~nm}^{6}, 8 \pi \nu n / 3=0.45 \times 10^{-24}\right.$ for the highest density [17-19]). Therefore, in this case $c_{3}$ cannot be used for an experimental determination of $\nu$.

By fitting a straight line to the experimental data in Fig. 2 and by using a value for $\nu$ obtained as described below, we find $B=(13 \pm 1) \times 10^{-24} \mathrm{~J} \mathrm{~nm}^{6}$, which is

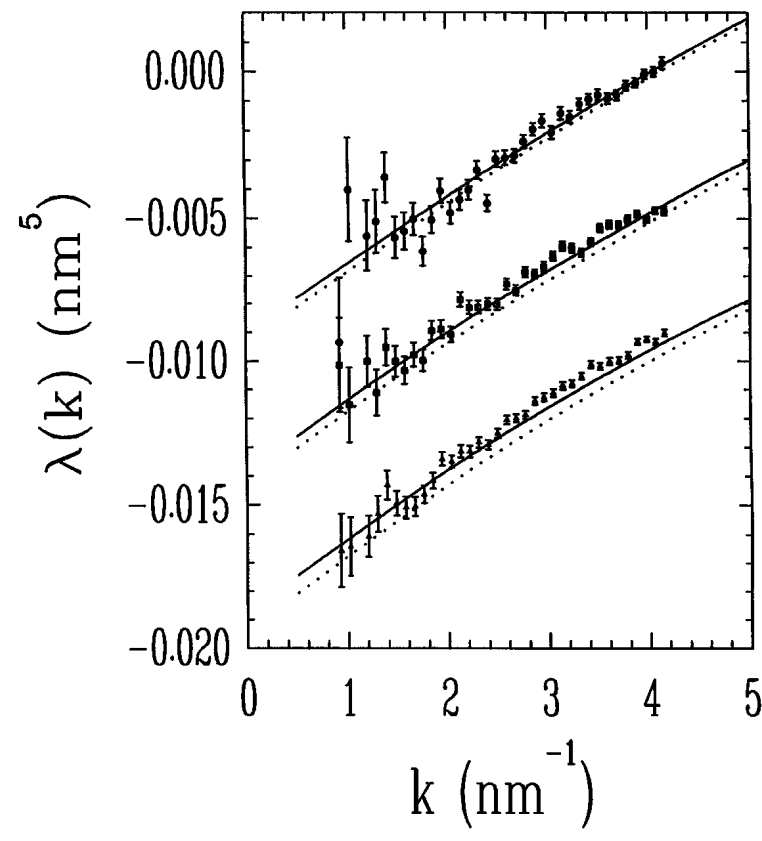

FIG. 2. Experimental results for $\lambda(k)$ [see Eq. (11)] for $n=$ $1.52,1.97,2.42 \mathrm{~nm}^{-3}$, respectively, reading from top to bottom (1.97 and $1.52 \mathrm{~nm}^{-3}$ results have been shifted upwards by 0.005 and 0.010 , respectively). The dotted line in these figures is based on the Aziz pair potential calculations reported in Ref. [17], while the full line includes also the Axilrod-Teller term. Note that for the highest $k$ values the calculated lines show a slight curvature.

in good agreement with the previous estimates given in the literature [18,19], which are $B=(12.7 \pm 0.9)$ and $(12.4 \pm 0.1) \times 10^{-24} \mathrm{~J} \mathrm{~nm}^{6}$, respectively. This confirms that for krypton low- $k S(k)$ measurements can lead to the experimental determination of the London dispersion energy.

We note that the theoretical curves in Fig. 2 are calculated with $\nu=2.23 \times 10^{-26} \mathrm{~J} \mathrm{~nm}^{9}$ as reported in literature [17-19]. Since inclusion of the AT potential in the calculation of $c(k)$ has a significant effect on $c_{2}$ (see Fig. 2), this coefficient should be used for the experimental determination of $\nu$. Since there is no analytical expression available for $c_{2}$, we used a different procedure. A least-squares fit of the theoretical lowdensity expansion [17] was made to $\lambda_{\exp }(k)$ for each of the three densities separately by varying $\nu$. The average yielded $\nu=(2.4 \pm 1.2) \times 10^{-26} \mathrm{~J} \mathrm{~nm}^{9}$. Although the value of $\nu$ we derive from our measurements is poorly defined, it is consistent with previous estimates of that quantity $[18,19]$. The accepted values of $B$ and $\nu$ were determined from dipole sum rules using a combination of several experimental and/or theoretical data. In principle the technique described in this Letter is unique [7] in that it allows direct experimental access to the long range part of the interaction potentials in an insulating fluid.

We would like to thank Dr. R. A. Robinson for help in providing the ${ }^{86} \mathrm{Kr}$, and Dr. J. Teixeira for helpful 
discussions and assistance in the use of the PAXE instrument. C. B. and P. E. would like to thank N.S.E.R.C. of Canada for financial support of their contribution to this project. Laboratoire Léon Brillouin is a Laboratoire Commun CEA-CNRS.

[1] F. London, Z. Phys. 63, 245 (1930); Z. Phys. Chem. B 11, 222 (1030).

[2] G.C. Maitland, M. Rigby, E. B. Smith, and W.A. Wakeham, Intermolecular Forces (Clarendon Press, Oxford, 1981).

[3] J.E. Enderby, T. Gaskell, and N.H. March, Proc. Phys. Soc. London 85, 217 (1965).

[4] R. Evans and T. J. Sluckin, J. Phys. C 14, 2569 (1981).

[5] I. L. McLaughlin and W.H. Young, J. Phys. C 15, 1121 (1982).

[6] R. J. Bowles and M. Silbert, J. Phys. C 17, 207 (1984).

[7] L. Reatto and M. Tau, J. Phys. Condens. Matter 4, 1 (1992).

[8] F. Barocchi, M. Zoppi, and P. A. Egelstaff, Phys. Rev. A 31, 2732 (1985).
[9] H. Fredrikze, J. B. van Tricht, A. A. van Well, R. Magli, P. Chieux, and F. Barocchi, Phys. Rev. Lett. 62, 2612 (1989).

[10] F. Barocchi, P. Chieux, H. Fredrikze, and R. Magli, Physica (Amsterdam) 180B-181B, 877 (1992).

[11] R. Magli, F. Barocchi, P. Chieux, and R. Fontana, Phys. Rev. Lett. 77, 846 (1996).

[12] R. M. Axilrod and E. Teller, J. Chem. Phys. 11, 299 (1943).

[13] B. P. Terburg, P. Verkerk, E. Jericha, and M. Zawisky, Nucl. Instrum. Methods Phys. Res., Sect. A 324, 247 (1993).

[14] A. Teitsma and P. A. Egelstaff, Phys. Rev. A 21, 367 (1980).

[15] N. J. Trappeniers, T. Wassenaar, and G. J. Wolkers, Physica (Utrecht) 32, 1503 (1966); J. Juza and O. Šifner, Acta Techn. CSAV 1, 1 (1976).

[16] G. Casanova, R. J. Dulla, D. A. Jonah, J. S. Rowlinson, and G. Saville, Mol. Phys. 18, 589 (1970).

[17] M. Tau, L. Reatto, R. Magli, P. A. Egelstaff, and F. Barocchi, J. Phys. Condens. Matter 1, 7131 (1989).

[18] J. M. Standard and P. R. Certain, J. Chem. Phys. 83, 3002 (1985).

[19] A. Kumar and W. J. Meath, Mol. Phys. 54, 823 (1985). 